\title{
Sclerostin is differently immunolocalized in metaphyseal trabecules and cor- tical bones of mouse tibiae
}

\author{
Tomoka Hasegawa ${ }^{1}$, Norio Amizuka ${ }^{1}$, Tamaki Yamada ${ }^{1}$, Zhusheng Liu ${ }^{1}$, Yukina Miyamoto ${ }^{1}$, Tomomaya \\ Yamamoto', Muneteru Sasaki ${ }^{1}$, Hiromi Hongo ${ }^{1}$, Reiko Suzuki ${ }^{1}$, Paulo Henrique Luiz de Freitas ${ }^{2}$, Tsuneyuki \\ YАмамото ${ }^{1}$, Kimimitsu OdA ${ }^{3}$, and Minqi $\mathrm{LI}^{4}$ \\ ${ }^{1}$ Department of Developmental Biology of Hard Tissue, Graduate School of Dental Medicine, Hokkaido University, Sapporo, Japan; \\ ${ }^{2}$ Department of Oral and Maxillofacial Surgery, Dr. Mário Gatti Municipal Hospital, Campinas, Brazil; ${ }^{3}$ Division of Biochemistry, \\ Niigata University Graduate School of Medical and Dental Sciences, Niigata, Japan; and ${ }^{4}$ Shandong Provincial Key Laboratory of Oral \\ Biomedicine, The School of Stomatology, Shandong University, Jinan, China
}

(Received 18 April 2013; and accepted 27 April 2013)

\begin{abstract}
Sclerostin, an osteocyte-derived molecule, has been reported to serve as a negative regulator of osteoblastic activity as well as bone remodeling. However, there is no report that verified the regional difference for sclerostin synthesis, and in this study we have investigated immunolocalization of sclerostin by comparing dentin matrix protein (DMP) 1, an osteocyte-derived factor broadly expressed in tibial metaphyses and cortical bone. In metaphyseal primary trabecules, a site of bone modeling, strong DMP1-reactivity was observed in osteocytic lacunar-canalicular system (OLCS), while faint staining for sclerostin was visible only in a few osteocytes. In secondary trabecules, in which bone remodeling begins, some osteocytes showed intense sclerostin-immunopositivity, though there were many DMP1-positive osteocytes. In cortical bone, there were more osteocytes reactive for sclerostin, when compared with those in the secondary trabecules. Silver impregnation verified that immature, primary trabecules contained randomly-oriented OLCS, while mature, cortical bone showed geometrically well-arrangement of OLCS. Taken together, though DMP1 is broadly synthesized in bone, sclerostin appears to be abundantly synthesized in regular OLCS of cortical bone, but less produced in irregular OLCS as seen in primary trabecules, indicating the regional difference for sclerostin synthesis.
\end{abstract}

Osteocytes embedded in mineralized bone matrix are the most abundant cells in bone, and extend their thin cytoplasmic processes through narrow passageway referred to as osteocytic canaliculi. Osteocytes establish the cellular network through gap junction of their cytoplasmic processes, by which they communicate not only neighboring osteocytes

Address correspondence to: Minqi Li, DDS, Ph.D Professor, Shandong Provincial Key Laboratory of Oral Biomedicine, The School of Stomatology, Shandong University, Wenhua West Road 44-1, Jinan 250012, China

Tel \& Fax: +86-531-88382095

E-mail: 1i_min_qi@hotmail.com but also osteoblasts and bone lining cells on bone surface $(1,5,6)$. Therefore, osteocytic lacunar-canalicular system (OLCS) is regarded as a functional syncytium. Meanwhile, each part of long bone might have its own function: 1) primary trabecule is a site for bone modeling based on mineralized cartilage cores during endochondral ossification (3), 2) secondary trabecule begins bone remodeling, and 3) cortical bone is a mature, well-mineralized bone which composes the outer wall of the long bone. The distribution patterns and structures of OLCS differ depending on such regional difference of bone. We have recently demonstrated that immature, primary trabecules contained randomly-oriented OLCS, while mature, cortical bone showed geomet- 
rically well arrangement of OLCS (9). We also defined that, by means of bone remodeling, the distribution of OLCS becomes progressively more regular as the individual grows (19).

Dentin matrix protein (DMP) 1 was originally identified in rat incisor's pulp cDNA library (8), and shown to be broadly expressed among osteocytes $(17,18)$. Due to its high calcium ion-binding capacity (18), DMP1 has been assumed to play a role in bone mineralization: a lack of DMP1 gave rise to rickets or osteomalacia in mice (11). This implies a pivotal role of osteocytes mediating DMP1 appears to be local regulation of mineralization. Meanwhile, sclerostin encoded by the SOST gene is another osteocyte-derived glycoprotein (22), which antagonizes Wnt signaling and increases $\beta$-catenin degradation, consequently inhibiting osteoblastic activities by passing through the osteocytic canaliculi $(12,21)$. Therefore, sclerostin has been considered as a negative regulator of osteoblastic bone formation as well as bone remodeling $(14,16,20,22)$. Yet, there is no report that verified the regional difference for sclerostin synthesis, e.g., in metaphyseal trabecular bone and the cortical bone. It seems possible that geometrical regularity of OLCS may affect the sclerostin synthesis.

Our aim of this study is to investigate immunolocalization of sclerostin in different areas, i.e., metaphyseal primary and secondary trabecules and cortical bone. For the aid of immunolocalization of sclerostin, we also examined DMP1, using multiple staining of alkaline phosphatase (ALPase) and tartrate-resistant acid phosphatase (TRAPase), hallmarks for osteoblastic and osteoclastic cells, respectively.

\section{MATERIALS AND METHODS}

Tissue preparation. We used eight 7-week old male ICR mice (Japan CLEA, Tokyo, Japan), following the principles for care and research use of animals set by Hokkaido University. Mice were anesthetized with an intraperitoneal injection of chloral hydrate and perfused with $4 \%$ paraformaldehyde diluted in $0.1 \mathrm{M}$ phosphate buffer ( $\mathrm{pH}$ 7.4) through the cardiac left ventricle. Tibiae were removed and immersed with the same fixative for $12 \mathrm{~h}$ at $4^{\circ} \mathrm{C}$. Samples were decalcified with $10 \%$ ethylenediamine tetraacetic disodium salt solution prior to embedding in OCT compound.

Histochemisty for sclerostin or DMP1 with multiple staining of alkaline phosphatase (ALPase) and tartrate-resistant acid phosphatase (TRAPase). Cryo- stat sections of tibiae were pretreated for inhibition of endogenous peroxidase activity with methanol containing $0.3 \%$ hydrogen peroxidase for $30 \mathrm{~min}$, and then, with $1 \%$ bovine serum albumin in PBS for $30 \mathrm{~min}$. These sections were incubated with goat anti mouse sclerostin antibody (R\&D systems, Inc., Minneapolis, MN) at a dilution of $1: 100$, or rabbit antibody against DMP1 (Takara Bio Inc., Otsu, Japan) at a dilution of $1: 500$. The sections thus treated were reacted with horseradish peroxidase (HRP)conjugated anti-goat IgGs (American Qualiex, San Clemente, CA) or HRP-conjugated anti-rabbit IgG (Jackson Immunoresearch Laboratories Inc., West Grove, PA) at a dilution of $1: 100$ at room temperature. Immune complexes were visualized using 3,3'-diaminobenzidine tetrahydrochloride (Dojindo Laboratories, Kumamoto, Japan).

After the immunostaining, detection of enzyme activities of ALPase and TRAPase was performed as previously reported (19). In brief, the immunostained sections were incubated with an aqueous solution containing $2.5 \mathrm{mg}$ of naphthol AS-BI phosphate (Sigma, St. Louis, MO) and $18 \mathrm{mg}$ of fast blue RR salt (Sigma) diluted in $30 \mathrm{~mL}$ of a $0.1 \mathrm{M}$ Tris- $\mathrm{HCl}$ buffer $(\mathrm{pH} 8.5)$ for $15 \mathrm{~min}$ at $37^{\circ} \mathrm{C}$. Then, theses slides were incubated in a mixture of $2.5 \mathrm{mg}$ of naphthol AS-BI phosphate, $18 \mathrm{mg}$ of red violet LB (Sigma) salt and $100 \mathrm{mM} \mathrm{L}(+)$ tartaric acid $(0.76 \mathrm{~g})$ diluted in $30 \mathrm{~mL}$ of a $0.1 \mathrm{M}$ sodium acetate buffer (pH 5.0) for $15 \mathrm{~min}$ at $37^{\circ} \mathrm{C}$. Counter staining was performed with methyl green.

Silver impregnation. We performed silver impregnation according to our previous report (13). In brief, the dewaxed sections were soaked in a $1 \%$ Protargol-S solution diluted in borax-boric acid ( $\mathrm{pH} 7.4)$ for $12-48 \mathrm{~h}$ at $37^{\circ} \mathrm{C}$. After rinsing with distilled water, the reaction was enhanced by an aqueous solution containing $0.2 \%$ hydroquinone, $0.2 \%$ citric acid and $0.7 \%$ nitric silver. After additional rinsing, the sections were reduced for $5 \mathrm{~min}$ with a solution of $2.5 \%$ anhydrous sodium sulfite, $0.5 \%$ potassium bromide and $0.5 \%$ amidol diaminophenol dihydrochloride. They were then treated with $1 \%$ gold chloride, and subsequently reduced by $2 \%$ oxalic acid amidol until visualizing black staining of osteocytic canaliculi. After rinsing with distilled water, sections were faintly stained with hematoxylene and eosin.

Statistical analysis for the percentage of DMP1-reactive, or sclerostin-positive osteocytes in metaphysis and cortical bone. The percentage of DMP1-reactive, or sclerostin-positive osteocytes in metaphyseal 
region and cortical bone was examined as described (19). The number of DMP1- or sclerostin-positive osteocytes was divided by the total number of osteocytes in the respective regions. All values are presented as means \pm standard deviation. Differences among groups were assessed by an unpaired Student's t-test, and considered statistically significant when $P<0.05$.

RT-PCR for evaluating expression of GAPDH, DMP1 and sclerostin. To evaluate gene expression of sclerostin and DMP1, total RNA was extracted from either cortical bone or metaphyseal trabecules. Firststrand cDNA synthesis was performed with SuperScript VILO cDNA Synthesis Kit (Life Technologies Co., Carlsbad, CA). The sequences for primers used are given in Table 1. The RT-PCR was performed using a thermal cycler as follows: denature at $94^{\circ} \mathrm{C}$

Table 1 The sequence for primers

\begin{tabular}{lll}
\hline mouse & Forward & TGTCTTCACCACCATGGAGAAGG \\
\cline { 2 - 3 } GAPDH & Revese & GTGGATGCAGGGATGAGTTGG \\
\hline mouse & Forward & CCCAGAGGCACAGGCAAATA \\
\cline { 2 - 3 } DMP1 & Revese & TCCTCCCCACTGTCCTTCTT \\
\hline mouse & Forward & GACCTCCCCACCATCCCTAT \\
\cline { 2 - 3 } sclerotin & Revese & GCGGAAATCCGGTCCGTT \\
\hline
\end{tabular}

for $30 \mathrm{~s}$, annealing at $60^{\circ} \mathrm{C}$ for $30 \mathrm{~s}$, extension at $72^{\circ} \mathrm{C}$ for $30 \mathrm{~s}$, and a final incubation at $72^{\circ} \mathrm{C}$ for $10 \mathrm{~min}$. RT-PCR products were subjected to $1.5 \%$ agarose gel electrophoresis, stained with ethidium bromide, and detected using E-Gel Imager (Life Technologies)

\section{RESULTS}

The irregularly-distributed osteocytes elongated their cytoplasmic processes in all direction in the primary trabecules of tibial metaphyses, in which cartilage cores interrupted the connection of their cytoplasmic processes (Fig. 1A). In the secondary trabecules, osteocytes tended to extend their cytoplasmic processes perpendicularly to the bone surface, forming relative regular OLCS (Fig. 1B). The cortical bone demonstrated more regular distribution of OLCS: flattened osteocytes parallel to the bone surface extended their cytoplasmic processes perpendicularly to the bone surface (Fig. 1C).

Immunohistochemical examination demonstrated an intense immunoreactivity of DMP1 throughout the metaphyseal primary trabecules, in which many ALPase-positive osteoblasts and TRAPase-reactive osteoclasts were seen (Fig. 2A). However, a small number of sclerostin-positive osteocytes was scat-
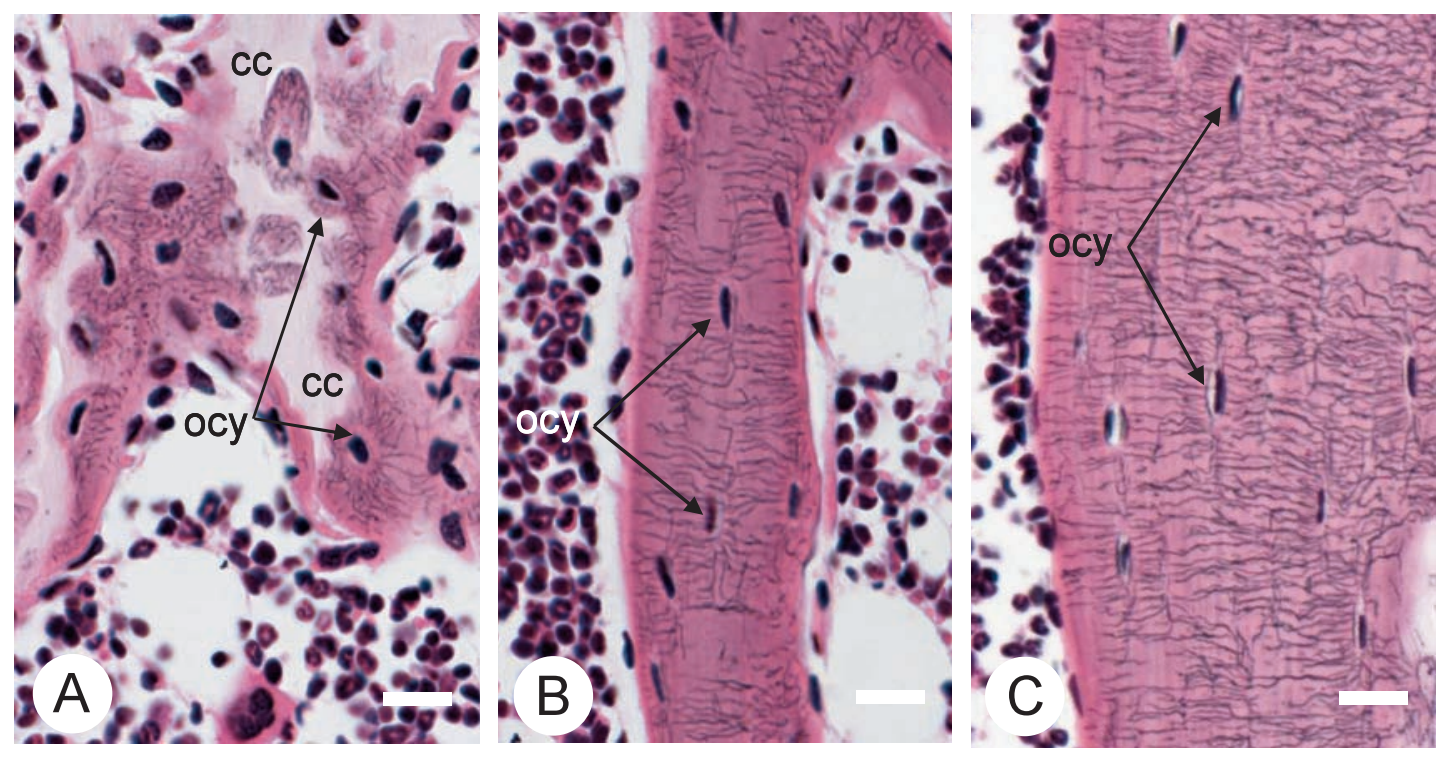

Fig. 1 Silver impregnation representing the distribution of osteocytic lacunar-canalicular system (OLCS). Metaphyseal primary trabecules show the irregular distribution of osteocytes (ocy), which elongate their cytoplasmic processes in all direction (A). The connection of the cytoplasmic processes of osteocytes is interrupted by the presence of inner cartilage cores (cc). In the secondary trabecule, osteocytes (ocy) are relatively arranged in a regular way, extending their cytoplasmic processes perpendicularly to the bone surface (B). Panel $\mathbf{C}$ shows more regular distribution of OLCS in cortical bone. The longitudinal axis of osteocytes (ocy) is parallel to the bone surface, and well-arranged cytoplasmic processes extend perpendicularly to the bone surface. Bar, $30 \mu \mathrm{m}$ 
tered in the corresponding area (Fig. 2B). At a higher magnification, strong DMP1-reactivity was observed in osteocytes and their canaliculi, while faint staining for sclerostin was visible only in a few osteocytes (Compare Figs. 2C and D). In the secondary trabecules, some osteocytes showed intense sclerostin-immunopositivity, though there were still many DMP1-reactive osteocytes and their canaliculi (Figs. 3A, B). In cortical bone, there were more osteocytes and their canaliculi positive for sclerostin, when compared with those in the secondary trabecules (Compare Figs. 3B and D). DMP1positive osteocytes were still many, but they seemed to have a similar population in both metaphyseal trabecules and the cortical bone (Fig. 3C). The cortical bone surface was covered with flattened osteoblasts weakly positive for ALPase, i.e., bone lining cells.

RT-PCR demonstrated less intense band indicative of sclerostin expression in metaphyseal region, com-
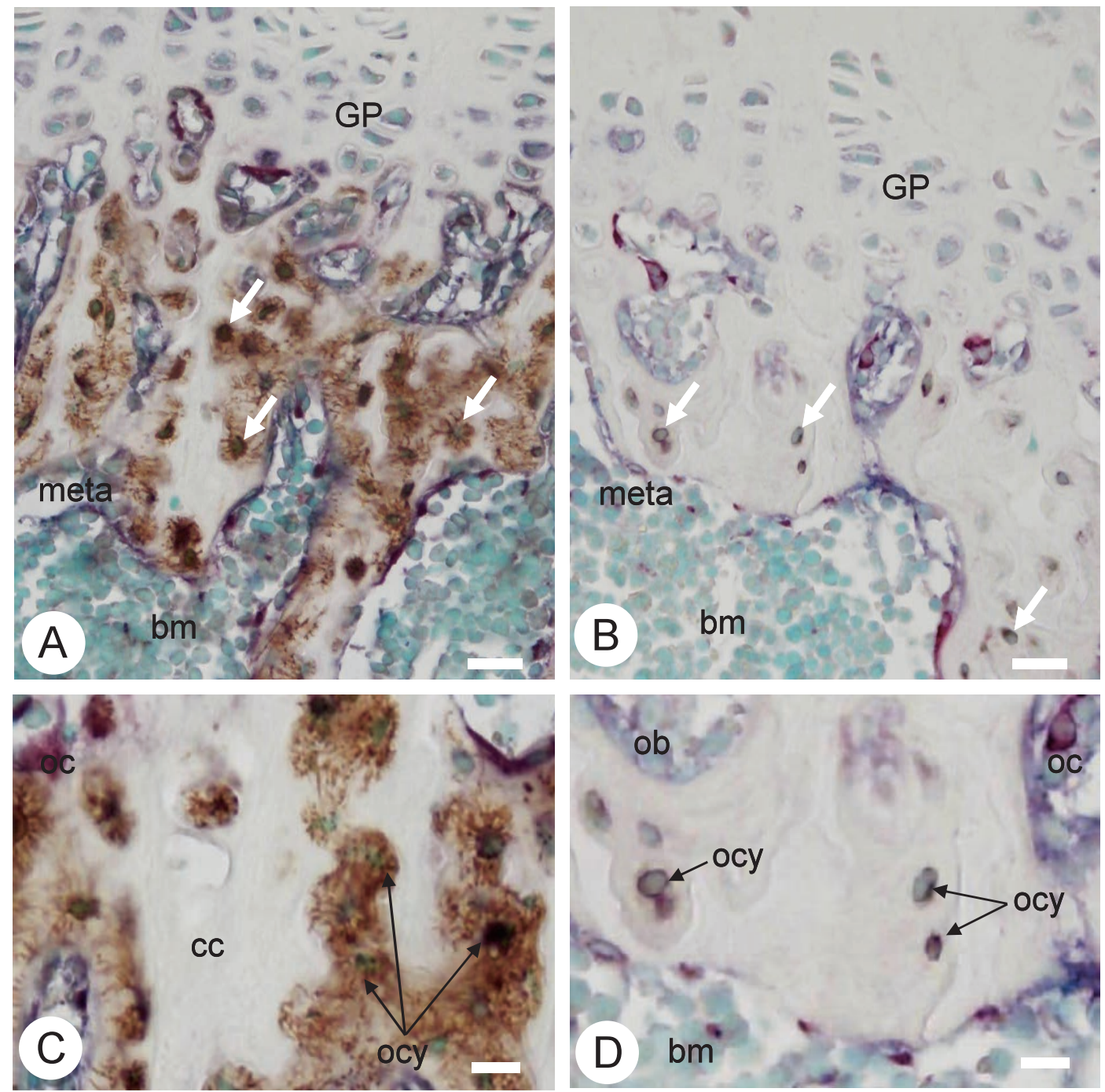

Fig. 2 Immunolocalization of DMP1 and sclerostin in multiple staining for ALPase and TRAPase in primary trabecules. Panels A and B show DMP1- and sclerostin-immunoreactivity (brown color), respectively, in multiple staining of ALPase (violet color) and TRAPase (red) in metaphyseal primary trabecules of tibiae. Many osteocytes (arrows) and their canaliculi with intense reactivity of DMP1 (brown color) are seen throughout primary trabecules (A), while osteocytes weakly positive for sclerostin (arrows, brown) are scattered (B). Panels $\mathbf{C}$ and D demonstrate higher magnifications of the primary trabecules for DMP1 (C) and sclerostin (D) staining. Note that DMP1 (brown color) is seen not only in osteocytes (ocy) but also their canaliculi (C), whereas faint sclerostin immunoreactivity is seen merely in osteocytes (ocy, D). There are many ALPasepositive osteoblasts (ob, violet color) and TRAPase-reactive osteoclasts (oc, red) in this region. GP: growth plate, meta: metaphysis, bm: bone marrow. Bar, A, B: $30 \mu \mathrm{m}, \mathrm{C}, \mathrm{D}: 10 \mu \mathrm{m}$ 

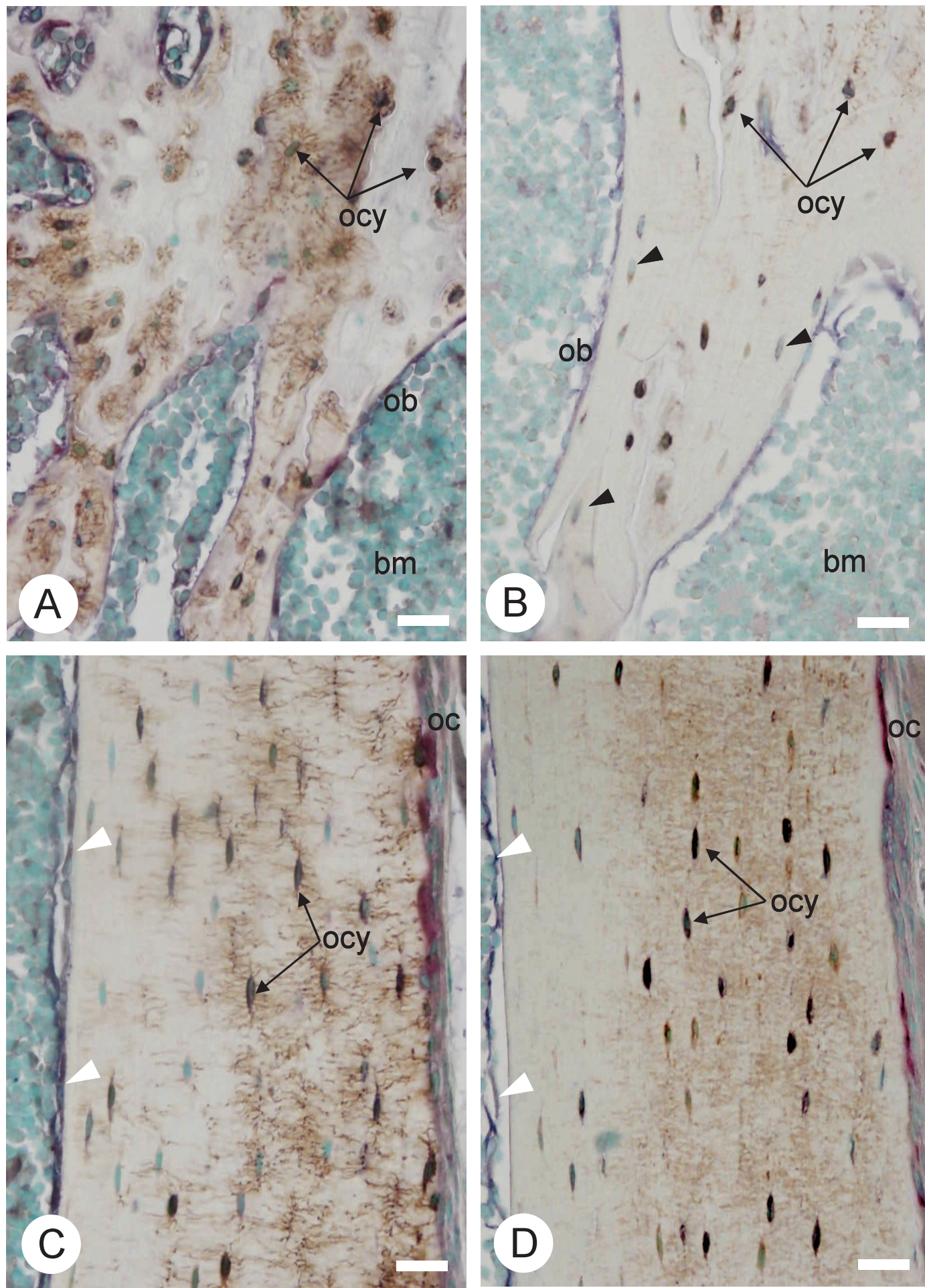

Fig. 3 Immunolocalization of DMP1 and sclerostin in multiple staining for ALPase and TRAPase in secondary trabecules and cortical bone. In the regions of secondary trabecule $(\mathbf{A}, \mathbf{B})$, osteoblasts (ob, violet color) are relatively flattened with weak ALPase-positivity. Intense reactivity for DMP1 (brown color) is still discernible in many osteocytes (ocy) and their canaliculi (A). Some osteocytes show an intense labeling for sclerostin (ocy, arrows), while many osteocytes display weak staining of sclerostin (arrowheads) (B). In cortical bone (C, D), endosteal bone surface is covered with flattened osteoblasts (violet color, white arrowheads), i.e., bone lining cells, while the periosteal surface shows some osteoclasts (oc, red). Note most osteocytes (ocy) are localized parallel to the endosteal bone surface. Many osteocytes reveal intense positivity for sclerostin (D) and DMP1 is still abundantly expressed in osteocytes and their canaliculi (C). bm: bone marrow. Bar, $30 \mu \mathrm{m}$ 


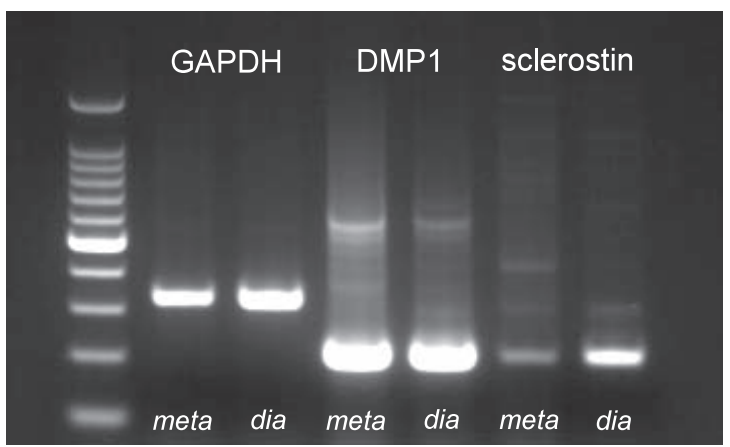

A

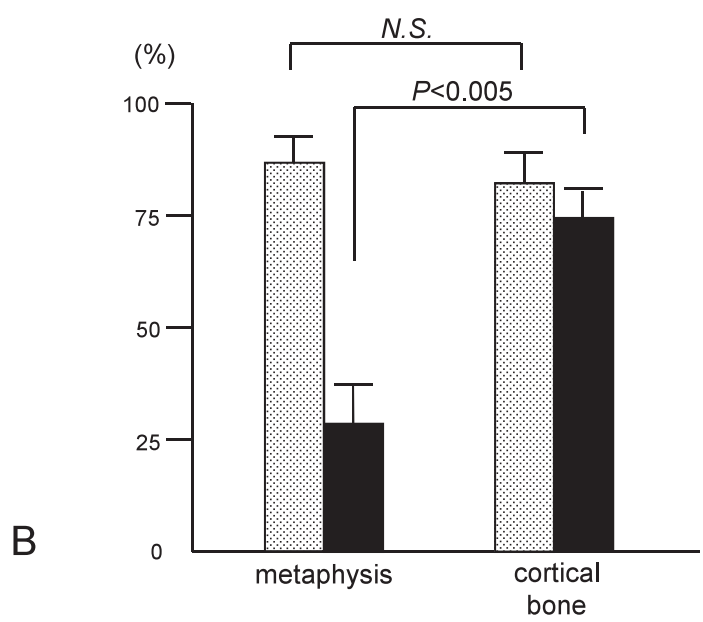

Fig. 4 RT-PCR evaluating expression of DMP1 and sclerostin, and statistical analysis for the index of DMP1-positive or sclerostin-reactive osteocytes. RT-PCR demonstrates less intense PCR product indicative of sclerostin expression in metaphyseal region (meta), compared with that in diaphyseal cortical bone (dia) (A). Notice no difference in intensity of PCR product indicative of DMP1 mRNA between metaphyseal and cortical bone. Panel B represents for the percentage of DMP1-positive (dotted box) and sclerostin-reactive (black colored box) osteocytes in the metaphysis and cortical bone. Statistical analysis on the index is presented as means \pm standard deviation. There is significant difference on the value of sclerostin-positive osteocytes between metaphysis and cortical bone $(P<0.005)$, while no significant difference (N.S.) is seen on the index of DMP1-positive osteocytes between the metaphysis and cortical bone.

pared with that in cortical bone, while DMP1 expression was similar in the metaphyseal trabecules and cortical bone (Fig. 4A). Consistent with RT-PCR, statistical analysis for the percentage of sclerostinreactive osteocytes showed significantly higher index in cortical bone compared with that in the metaphysis $(74.05 \pm 8.71$ vs $27.05 \pm 10.37, P<$
0.005, Fig. 4B). These findings apparently suggest the regional difference in sclerostin synthesis by osteocytes.

\section{DISCUSSION}

The primary metaphyseal trabecule is the site in which many active osteoblasts deposit abundant bone matrices and rapidly differentiate into osteocytes (3). The geometrical regularity of osteocytes and the canalicular connection of neighboring osteocytes are completely disorganized, e.g., by inner cartilage cores, indicating less possibility of osteocytic function as a syncytium (19). We postulate that the primary trabecules may serve for making bony scaffolds for establishing subsequent secondary trabecules, and osteocytes in this region may hardly affect to osteoblastic activity. The present study demonstrated many active osteoblasts in the primary trabecules, leading to an idea that less expression of sclerostin allows active form of osteoblasts in this region. Unlike to sclerostin, osteocytes synthesized abundant DMP1 regardless with regional difference in bone, implicating that this molecule would not affect osteoblastic activity though it was reported to be involved in mineralization in bone (7). In contrast, cortical bone was shown to possess well-organized distribution of OLCS, indicative of functional syncytium. It seems reasonable that cortical bone with intense sclerostin-positive osteocytes was covered by bone lining cells, inactive osteoblasts with weak ALPase (Figs. 3C, D). We hypothesize that geometrically well-arranged OLCS in mature cortical bone would secret plenty of sclerostin inhibiting the cell activity of osteoblasts, which may possibly lessen the frequency of bone remodeling for maintaining maturity of the cortical bone. Thus, the regional difference of OLCS's regularity appears to affect osteocytic functions including sclerostin synthesis.

Recently, some investigators demonstrated that treatment with antibodies to sclerostin enhanced bone healing from osteoporosis $(2,11)$, and parathyroid hormone $(\mathrm{PTH})$ reduced the expression of sclerostin, resulting in osteoblastic activation and consequent bone formation $(4,10,16)$. But, our findings in this study may suggest that biological effects for sclerostin antibody and PTH treatment would differ depending on each region of bone. We have reported that osteoprotegerin-deficient mice bearing high bone turnover reduced the synthesis of sclerostin, which, however, appeared to be dependent of the region of bone (13). In addition, sclerostin 
levels were shown to be a finely tuned mechanism by osteocytes in response to mechanical stimulation (15); possibly, osteocytes regularly-distributed in cortical bone would be easy to sense mechanical stress for secreting sclerostin. Although few studies have paid attention for immunolocalization of sclerostin, histochemical findings in our study may be helpful for comprehension for local effects in bone after administration of PTH and sclerostin antibodies.

\section{Acknowledgements}

This study was partially supported by grants from National Nature Science Foundation of China $(\mathrm{Li}$, No.81271965) and Japanese Society for the Promotion of Science (Amizuka N, Suzuki R). Tomoka Hasegawa and Hiromi Hongo are research fellows for young scientists, Japan Society for Promotion of Science.

\section{REFERENCES}

1. Aarden EM, Burger EH and Nijweide PJ (1994) Function of osteocytes in bone. J Cell Biochem 55, 287-299.

2. Agholme F, Li X, Isaksson H, Ke HZ and Aspenberg P (2010) Sclerostin antibody treatment enhances metaphyseal bone healing in rats. $J$ Bone Miner Res 25, 2412-2418.

3. Amizuka N, Hasegawa T, Oda K, Luiz de Freitas PH, Hoshi K, Li M and Ozawa H (2012) Histology of epiphyseal cartilage calcification and endochondral ossification. Front Biosci 4, 2085-2100.

4. Bellido T, Ali AA, Gubrij I, Plotkin LI, Fu Q, O'Brien CA, Manolagas SC and Jilka RL (2005) Chronic elevation of parathyroid hormone in mice reduces expression of sclerostin by osteocytes: a novel mechanism for hormonal control of osteoblastogenesis. Endocrinology 146, 4577-4583.

5. Donahue HJ (2000) Gap junctions and biophysical regulation of bone cell differentiation. Bone 26, 417-422.

6. Doty SB (1981) Morphological evidence of gap junctions between bone cells. Calcif Tissue Int 33, 509-512.

7. Feng JQ, Ward LM, Liu S, Lu Y, Xie Y, Yuan B, Yu X, Rauch F, Davis SI, Zhang S, Rios H, Drezner MK, Quarles LD, Bonewald LF and White KE (2006) Loss of DMP1 causes rickets and osteomalacia and identifies a role for osteocytes in mineral metabolism. Nat Genet 38, 1310-1315.

8. George A, Gui J, Jenkins NA, Gilbert DJ, Copeland NG and Veis A (1994) In situ localization and chromosomal mapping of the AG1 (Dmp1) gene. J Histochem Cytochem 42, 15271531.

9. Hirose S, Li M, Kojima T, de Freitas PH, Ubaidus S, Oda K, Saito C and Amizuka N (2007) A histological assessment on the distribution of the osteocytic lacunar canalicular system using silver staining. J Bone Miner Metab 25, 374-382.
10. Kramer I, Keller H, Leupin O and Kneissel M (2010) Does osteocytic SOST suppression mediate PTH bone anabolism? Trends Endocrinol Metab 21, 237-244.

11. Lewiecki EM (2011) Sclerostin monoclonal antibody therapy with AMG 785: a potential treatment for osteoporosis. Expert Opin Biol Ther 11, 117-127.

12. Li X, Zhang Y, Kang H, Liu W, Liu P, Zhang J, Harris SE and $\mathrm{Wu} \mathrm{D}$ (2005) Sclerostin binds to LRP5/6 and antagonizes canonical Wnt signaling. $J$ Biol Chem 280, 19883-19887.

13. Masuki H, Li M, Hasegawa T, Suzuki R, Ying G, Zhusheng L, Oda K, Yamamoto T, Kawanami M and Amizuka N (2010) Immunolocalization of DMP1 and sclerostin in the epiphyseal trabecule and diaphyseal cortical bone of osteoprotegerin deficient mice. Biomed Res 31, 307-318.

14. Poole KE, van Bezooijen RL, Loveridge N, Hamersma H, Papapoulos SE, Löwik CW and Reeve J (2005) Sclerostin is a delayed secreted product of osteocytes that inhibits bone formation. FASEB J 19, 1842-1844.

15. Robling AG, Niziolek PJ, Baldridge LA, Condon KW, Allen MR, Alam I, Mantila SM, Gluhak-Heinrich J, Bellido TM, Harris SE and Turner CH (2008) Mechanical stimulation of bone in vivo reduces osteocyte expression of Sost/sclerostin. $J$ Biol Chem 283, 5866-5875.

16. Silvestrini G, Ballanti P, Leopizzi M, Sebastiani M, Berni S, Di Vito M and Bonucci E (2007) Effects of intermittent parathyroid hormone (PTH) administration on SOST mRNA and protein in rat bone. $J$ Mol Histol 38, 261-269.

17. Tatsumi S, Ishii K, Amizuka N, Li M, Kobayashi T, Kohno K, Ito M, Takeshita S and Ikeda K (2007) Targeted ablation of osteocytes induces osteoporosis with defective mechanotransduction. Cell Metab 5, 464-475.

18. Toyosawa S, Shintani S, Fujiwara T, Ooshima T, Sato A, Ijuhin N and Komori T (2001) Dentin matrix protein 1 is predominantly expressed in chicken and rat osteocytes but not in osteoblasts. $J$ Bone Miner Res 16, 2017-2026.

19. Ubaidus S, Li M, Sultana S, de Freitas PH, Oda K, Maeda T, Takagi R and Amizuka N (2009) FGF23 is mainly synthesized by osteocytes in the regularly distributed osteocytic lacunar canalicular system established after physiological bone remodeling. J Electron Microsc (Tokyo) 58, 381-392.

20. van Bezooijen RL, Roelen BA, Visser A, van der Wee-Pals L, de Wilt E, Karperien M, Hamersma H, Papapoulos SE, ten Dijke P and Löwik CW (2004) Sclerostin is an osteocyte-expressed negative regulator of bone formation, but not a classical BMP antagonist. J Exp Med 199, 805-814.

21. Veverka V, Henry AJ, Slocombe PM, Ventom A, Mulloy B, Muskett FW, Muzylak M, Greenslade K, Moore A, Zhang L, Gong J, Qian X, Paszty C, Taylor RJ, Robinson MK and Carr MD (2009) Characterization of the structural features and interactions of sclerostin: molecular insight into a key regulator of Wnt-mediated bone formation. $J$ Biol Chem 284, 10890-10900.

22. Winkler DG, Sutherland MK, Geoghegan JC, Yu C, Hayes T, Skonier JE, Shpektor D, Jonas M, Kovacevich BR, StaehlingHampton K, Appleby M, Brunkow ME and Latham JA (2003) Osteocyte control of bone formation via sclerostin, a novel BMP antagonist. EMBO J 22, 6267-6276. 